Background: Krebs von den Lungen-6 (KL-6) was recently found to be a serum biomarker for various disease associated interstitial lung disease (ILD) including primary Sjögren syndrome (pSS). ${ }^{1} \mathrm{KL}-6$ is a high-molecular-weight glycoprotein in the Mucin 1 protein group and is majorly expressed by regenerating type II pneumocytes; hence, serum KL-6 levels may reflect the severity of pulmonary damage with regeneration. ${ }^{2}$ Human $\mathrm{KL}-6$ also promotes the proliferation and survival of pulmonary fibroblasts and the differentiation of myofibroblasts, which enhance fibrosis. ${ }^{3,4}$

Objectives: To evaluate the agreement between the latex particle-enhanced turbidimetric immunoassay with enzyme-linked immunosorbent assay (ELISA) and association with clinical phenotypes

Methods: This retrospective case-control study included 39 patients with pSS, of whom $21(53.85 \%)$ patients developed ILD at the end of follow-up. The serum KL-6 level was compared between latex particle-enhanced turbidimetric immunoassay (Nanopia) and ELISA (MBS2601395; MyBioSource, CA, USA). Electronic medical records were reviewed, including clinical information, images, pulmonary function test, and laboratory results on inclusion, and a chest physician reviewed the results of pulmonary radiography.

Results: The two serum KL-6 immunoassays revealed a moderate correlation with a Pearson product-moment correlation coefficient of 0.427 . Serum KL-6 levels, measured using ELISA, were $1920.10 \pm 1974.26 \mathrm{U} / \mathrm{mL}$ and $894.11 \pm$ $788.53 \mathrm{U} / \mathrm{mL}$ in the ILD and non-ILD groups, respectively $(p=0.001)$. The latex particle-enhanced turbidimetric immunoassay for serum KL-6 was $459.62 \pm$ $331.41 \mathrm{U} / \mathrm{mL}$ and $265.33 \pm 105.37 \mathrm{U} / \mathrm{mL}$ in the ILD and non-ILD group, respectively $(p=0.074)$. The predictive values of serum KL- 6 in the area under the receiver-operating characteristic curve were 0.810 and 0.669 in ELISA and latex particle-enhanced turbidimetric immunoassay, respectively.

Conclusion: Serum KL-6 is a predicting biomarker in pSS patients who may develop ILD. However, the methodology of immunoassay may influence the efficacy of the prediction and clinical association.

References:

[1] Chiu Y-H, Lu C-C, Liu F-C, et al. FRI0228 KL-6 AS A BIOMARKER OF DEVELOPING INTERSTITIAL LUNG DISEASE IN PATIENTS WITH SJÖGREN SYNDROME. Ann Rheum Dis. 2019; 78: 793.

[2] Yousefi M, Dehghani S, Nosrati R, et al. Aptasensors as a new sensing technology developed for the detection of MUC1 mucin: A review. Biosensors \& bioelectronics. 2019; 130: 1-19.

[3] Xu L, Yan DR, Zhu SL, et al. KL-6 regulated the expression of HGF, collagen and myofibroblast differentiation. Eur Rev Med Pharmacol Sci. 2013; 17 : 3073-7.

[4] Ohshimo S, Yokoyama A, Hattori N, Ishikawa N, Hirasawa $Y$ and Kohno N. $\mathrm{KL}-6$, a human MUC1 mucin, promotes proliferation and survival of lung fibroblasts. Biochem Biophys Res Commun. 2005; 338: 1845-52.

Acknowledgments: This work was supported by National Defense Medical Center and Tri-Service General Hospital (TSGH-D-109183). The authors thank Kuo's Yuan In Enterprise co., LTD for supporting Nanopia KL-6 Kit.

Disclosure of Interests: None declared

DOI: 10.1136/annrheumdis-2020-eular.1006

\section{AB1237 DIAGNOSTIC VALUE OF PROTEASOMAL AND AUTOPHAGIC MARKERS IN MUSCULAR DISEASES}

U. Drott ${ }^{1}$, P. Harter ${ }^{2}$, H. Burkhardt ${ }^{3}$, M. Mittelbronn ${ }^{4} .{ }^{1}$ Rheumatology Division, Goethe-University, Frankfurt, Germany; ${ }^{2}$ Institute of Neurology (Edinger Institute), Goethe University, Frankfurt, Germany; ${ }^{3}$ Rheumatology Division, Goethe-University Frankfurt, Frankfurt, Germany; ${ }^{4}$ Luxembourg Center of Neuropathology (LCNP), Dudelange, Germany

Background: Deficient cellular degradation pathways such as autophagy and the ubiquitin proteasome system (UPS) show a correlation with the onset of neurodegenerative diseases. Especially immune-mediated inflammatory myopathies often show therapy-resistant phenotypes with medical need for further understanding of pathogenesis und possible treatment.

Objectives: The aim of this work was to study an association of these two degradation pathways in a large group of different muscle entities and to examine a possible influence in the pathogenesis of the investigated muscle diseases. Furthermore, a potential benefit in diagnostics was studied using factors such as ubiquitin, p62, NBR1 and LC3 and their role as adapter molecules.

Methods: We examined ubiquitin, p62, NBR1 and LC3 on muscle biopsies from patients with the diagnosis of S-IBM, dermato- and polymyositis, muscular dystrophy, neurogenic atrophy, myotonic dystrophy type II (PROMM) and metabolic myopathies such as Pompe and McArdle disease. Immunohistochemical single and double stainings as well as immunofluorescence stainings were performed on cryosections. Furthermore, Western blot analysis was performed. The use of a histological score, defined as the product of the frequency of positive fibres and staining intensity, was used to investigate possible differences between the diseases in the expression of the investigated factors.
Results: Especially in s-IBM, myofibrillar myopathies and Pompe disease, proteasomal and autophagic markers were detected. On the other hand, neurogenic atrophy, McArdle disease and the majority of the myotonic dystrophies type I showed no positive signals, except for p62, which was detected especially in internalized nuclei and regenerative fibres. Overall, in all entities in regenerative and necrotic as well as in atrophic fibres, a positive staining for ubiquitin, p62, NBR1 and LC3 could be shown. Dermatomyositis showed a moderate immunoreactivity for p62 and NBR1 in the area of the perifascicular atrophy. Polymyositis showed a strong endomysial reaction in the area of the attacked muscle fibres, especially in the area of the lymphocytic infiltrates. The histological score showed that ubiquitin was significantly higher in s-IBM than in polymyositis. NBR1 was significantly higher in S-IBM than in dermatomyositis and muscular dystrophies. LC3 and p62 showed a significantly higher level in s-IBM as compared to dermatomyositis and polymyositis. Except for NBR1, a positive correlation of autophagic and proteasomal markers was shown in dermatomyositis. There was also a positive correlation between LC3 and ubiquitin in polymyositis. Using multivariate analysis and recursive partitioning, we could show a predictability of the diagnosis of S-IBM with a LC3 score above 3. On the other hand, a value below 3 excluded the diagnosis of S-IBM.

Conclusion: In particular, s-IBM, myofibrillar myopathies and Pompe disease showed a possible involvement disturbed proteasomal and autophagic degradation pathways in their pathogenesis. In addition, p62 and NBR1 seemed to have an important role in the immune response. Furthermore, the altered autophagic and proteasomal degradation pathways may be involved in ageing processes sarcopenia and disease. In particular, LC3 seems to be suitable as a screening marker to recognize an idiopathic inflammatory myopathy. The other markers, such as p62, LC3, and NBR1, would be able to distinguish between the subgroups of idiopathic inflammatory myopathies. Therefore, LC3 could be included as a marker in the routine of neuropathological diagnostics. Further studies are needed to fully understand the role of proteasomal and autophagic factors in possible immune suppressive and immune modulatory therapies and to assess possible side effects.

Disclosure of Interests: Ulrich Drott: None declared, Patrick Harter: None declared, Harald Burkhardt Grant/research support from: Pfizer, Roche, Abbvie Consultant of: Sanofi, Pfizer, Roche, Abbvie, Boehringer Ingelheim, UCB, Eli Lilly, Chugai, Bristol Myer Scripps, Janssen, and Novartis, Speakers bureau: Sanofi, Pfizer, Roche, Abbvie, Boehringer Ingelheim, UCB, Eli Lilly, Chugai, Bristol Myer Scripps, Janssen, and Novartis, Michel Mittelbronn: None declared DOI: 10.1136/annrheumdis-2020-eular.5897

\section{AB1238 THE ARABIC LUPUSPRO: CROSS-CULTURAL VALIDATION OF A DISEASE-SPECIFIC PATIENT- REPORTED OUTCOME TOOL FOR QUALITY OF LIFE IN LUPUS PATIENTS}

N. E. Elkaraly ${ }^{1}$, S. I. Nasef ${ }^{1}$, A. S. Omar ${ }^{1}$, A. M. Fouad ${ }^{2}$, J. Meenakshi ${ }^{3}$, A. E. Mohamed ${ }^{1} .{ }^{1}$ Faculty of Medicine, Suez Canal University, Physical Medicine, Rheumatology and Rehabilitation, Ismailia, Egypt; ${ }^{2}$ Faculty of Medicine, Suez Canal University, Public Health, Occupational and Environmental Medicine, Ismailia, Egypt; ${ }^{3}$ Rush University, Medicine and Behavioral Sciences, Chicago, IL, United States of America

Background: Systemic lupus erythematosus (SLE) is a chronic autoimmune disease with high morbidity and disability that affects all aspects of quality of life (QoL). ${ }^{(1)}$ LupusPRO is a disease-targeted patient-reported outcome tool for the measurement of health and non-health related quality of life (HRQoL and Non$\mathrm{HRQoL}$ ) in SLE patients. (2)

Objectives: To translate, cross-culturally adapt, and validate the LupusPRO v.1.8 into the Arabic Language.

Methods: LupusPRO v.1.8 was translated into Arabic following the for ward-backward translation guideline. (3) The Arabic version of LupusPRO was subsequently applied to 107 Egyptian SLE patients. To assess test-retest reliability, the Arabic LupusPRO was completed on two occasions, two weeks apart. Validation was performed against the Arabic version of Medical Outcomes Study Short Form (SF-36), the Systemic Lupus Erythematosus Disease Activity Index (SELENA-SLEDAI) and the Systemic Lupus Internationa Collaborating Clinics/American College of Rheumatology damage index (SDI) Discriminant validity of Arabic LupusPRO was evaluated against the SF-36 (item 1), while the conceptual framework was evaluated by the confirmatory factor analysis (CFA)

Results: The study included 107 patients, 95\% of whom were women. Their median age was 32 (range:18-55) years. Arabic LupusPRO's Cronbach's alpha ranged from 0.71 to 0.98 except for the social support domain (0.65). Test-retest reliability ranged from 0.83 to 0.98 . Convergent validity with corresponding domains of the SF-36 was satisfactory. Criterion validity showed a weak and non-significant correlation of lupusPRO domains with SDI except for physical domain and significant weak correlation with SELENA-SLEDAI (Table1). 